\title{
The Sound Medicine of Brian Dailey, M.D., F.A.C.E.P.
}

\section{Russ Mason, M.S.}

EDITOR'S NOTE: The use of sound and music for healing has a long history. In the 1950s, Robert Monroe pioneered a new kind of sound technology. This patented process used tones to create specific physiologic states within the brain and, thus, balance the electrical activity of both hemispheres of the brain. The combination of tones at different frequencies, delivered to each ear in specific patterns, is received and integrated by the brain in a way that promotes various states of healing, relaxation, concentration, focus, and intuition. The technology is available in the form of compact discs (CDs) produced at The Monroe Institute, in Faber, Virginia (see box entitled About the Monroe Institute). Today, health care practitioners are finding significant new ways to assist their patients with this innovative sound technology. Among them is Brian Dailey, M.D., F.A.C.E.P.

\section{Introduction}

B rian Dailey, M.D., F.A.C.E.P., is a clinical instructor in alternative and complementary medicine (ACM) therapies at the University of Rochester School of Medicine and Dentistry (Rochester, New York) and is board certified in emergency medicine. He is an attending physician in emergency medicine at Rochester General Hospital (Rochester, New York), a long-time practitioner of energy medicine, and a Reiki Master and teacher who uses crystal therapy and aromatherapy in his medical practice. He lectures and conducts workshops throughout the United States and Canada about the application of these modalities. He is actively involved in research involving consciousness exploration as well as energetic and remote healing.

In 1990, Dr. Dailey experienced The Monroe Institute's (TMI's) Hemi-Sync ${ }^{\circledR}$ technology in a weekend workshop conducted by Joseph Gallenberger, Ph.D., in Rochester, NY. Impressed and intrigued with what he had experienced, Dr. Dailey subsequently attended many of the residential courses offered at TMI. Further interest led to his membership in the professional division of TMI, a position on the Institute's advisory board, and membership in the Dolphin Energy Club (DEC), a group of remote healers. Additional experience convinced him of the therapeutic value of Hemi-Sync for his patients in the hospital, outpatient, and home environments.

\section{The Interview}

Russ Mason: Please tell me how you use sound to help your patients heal.

Brian Dailey: As a physician trained in both traditional and energetic medicine, I have found that sound may have a therapeutic effect for patients with a variety of illnesses, and that sound can be extremely effective in assisting healthy individuals with concentration, insight, intuition, creativity, and meditation.

RM: Please explain about the sounds specifically and what they do.

BD: Robert Monroe, founder of TMI, discovered that specific sound patterns and frequencies have beneficial, identifiable, and measurable effects on an individual's physical and mental functions. For example, some combinations of frequencies enhance alertness; others may induce sleep; and still others evoke expanded states of consciousness.

From this research Hemi-Sync CDs were produced at TMI to enhance relaxation and stress reduction; provide healing support for cancer treatment, surgery, hypertension, stroke, asthma, and many other applications. I use the technique myself for insight, intuition, improving my own health, and helping to engender a deeper spiritual connection. It has improved my intuitive insight into difficult-to-diagnose medical cases. It has vastly increased my abilities as an energy healer.

My patients have benefited as well. For example, TMI's Surgical Support Series showed in a clinical trial that patients who listened to Hemi-Sync during surgery required 4.5 times less anesthesia than either of the study's control groups-one that listened to blank tapes and one that listened to classical music. ${ }^{1}$ I encourage all my patients who are undergoing surgery to consider using the CDs, as listening can reduce their pain, their need for pain medication, allow them to awaken more quickly in recovery, and hasten discharge.

RM: How did you discover TMI and the Hemi-Sync recordings?

BD: I first learned about TMI because of my interest in remote viewing. The military used remote viewing to psychically obtain information not available by ordinary physical senses, often with great success. I found this intriguing, particularly the idea that remote viewing might be used to assist in medical diagnosis. The military had sent many of their remote viewers to TMI work- 
shops because the workshops enhanced the remote viewers' performance.

I also learned of people such as Barbara Brennan, Ph.D. (founder of the Barbara Brennan School of Healing in Miami, Florida), and Elizabeth Kubler-Ross, M.D., (based in Scottsdale, Arizona) who had been to TMI, and I was intrigued with the work that TMI was doing in consciousness research. TMI was a happening place!

One day a synchronicity occurred. A poster advertised TMI, and a local intensive weekend, with Dr. Gallenberger. The poster said that one could expand one's cognitive function with the use of sound. I attended the weekend intensive, and the experience just opened up, in a nearly explosive manner, my appetite for expanding my own awareness and intuition. The weekend provided a wonderful introduction to TMI and Hemi-Sync. It was a tasty appetizer, leaving me hungry for the main entrée.

RM: What, specifically, did you do in the weekend intensive?

BD: We listened to Hemi-Sync recordings that produced alpha and theta brainwave states. These induced profound relaxation and well-being. They also opened my insight and intuition and I was amazed at the ease with which it worked. Someone practicing meditation for 20 years might achieve a hemispheric theta

\begin{tabular}{|l|}
\hline To Contact \\
Dr. Brian Dailey \\
Brian Dailey, M.D., \\
F.A.C.E.P. \\
Rochester General Hospital \\
I425 Portland Avenue \\
Rochester, NY I462 I \\
Phone: (585) 922-4000 \\
e-mail: \\
bdailey@rochester.rr.com \\
Web site: \\
www.ViaHealth.org \\
\hline
\end{tabular}

brainwave state after several hours of meditating, and maintain it for 5-15 minutes. We were learning to do it in mere minutes-and maintaining it for long periods of time.

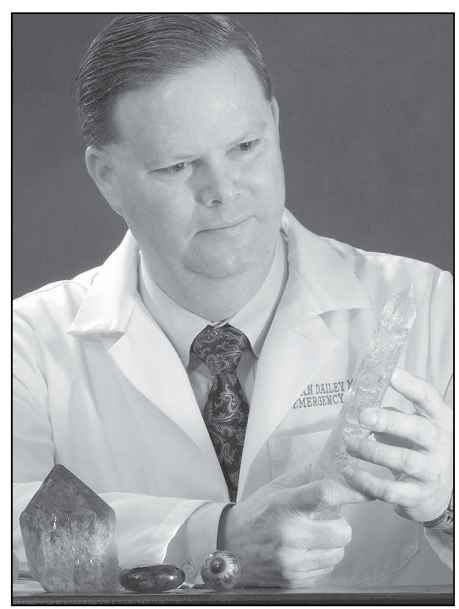

Brian Dailey, M.D., F.A.C.E.P., examines a healing crystal. Photo credit: Jeff Blackman, Manager of Vis ual Communications, Rochester General Hospital, Rochester, New York.

RM: Have any Buddhist meditators tried the products?

BD: Yes. A 92-year-old monk went to TMI and, after experi-

\section{Hemi-Sync ${ }^{\circledR}$ CD Use in Practice}

\section{Brief Case Reports}

\section{Childbirth and Breast Cancer}

Jackie G, a 34-weeks pregnant patient, developed a highly malignant breast cancer. Reiki, crystals, and Hemi Sync ${ }^{\circledR}$ recordings were used in addition to conventional care.

Meta Music was used to induce relaxation, and another series of recordings assisted her with childbirth and nursing.

"The sounds really helped reduce the discomfort of my labor," Jackie reported, as she gave birth to her son, after a 1-hour-and-20minute labor, with no episiotomy, and no repair.

Jackie used the Surgical Support Series for her 7-hour cancer surgery and experienced little need for pain medication postoperatively. She then used the Chemotherapy Companion to reduce the side-effects of chemotherapy and the Positive Immunity Series enhanced her immune function and well-being; as of today, she remains healthy.

\section{Injuries}

Kelly A, was in the surgical intensive care unit after a severe motor vehicle accident. She had sustained a severe liver laceration with anemia (her hematocrit $=27$ percent) as well as a compression fracture of her first lumbar vertebra, which had kept her bedridden for 3 days because of pain.

After her first Hemi-Sync and Reiki session, she was able to get out of bed and, on the fifth day, after four treatments, her hematocrit had risen to a normal level of 36 percent, the equivalent of a transfusion of three units of blood (without the associated high cost, or health risks associated with blood transfusion). She did not need Epogen injections.

\section{Cancer}

Gretchen G. was 21 when she was diagnosed with lymphoma. She was only able to complete 4 of her scheduled 12 chemotherapy regimens, because she had such pronounced nausea and vomiting, in addition to complete hair loss.
One (1) year later, Gretchen's tumor had quadrupled in size. Upon referral from her doctor, she came to Rochester, New York, for evaluation and treatment. She was initially extremely reluctant to undergo the chemotherapy her oncologist had recommended, based on her prior experience and her holistic beliefs.

We discussed Gretchen's misgivings, with the understanding that this chemotherapy was to make her well, and she liked the idea of having a "Love Potion" infused in her rather than something she thought of as toxic. When she had a severe episode of nausea and vomiting with her first episode of chemotherapy she was given the Chemotherapy Companion, and 45 minutes later, when she woke up, she was able to eat lunch.

Another patient, Ardith F., experienced a severe episode of nausea, vomiting, and diarrhea with chemotherapy 2 weeks before coming to Rochester. After listening to Chemotherapy Companion during her next chemotherapy treatment, and eating lunch during her infusion, she later remarked, "I feel better than when I came in."

Susan S., another patient with cancer, adapted the Resonant Energy Balloon (REBAL) concept from a CD in a new way. Here is how she described her experience:

They [tell] you to inhale and, as you exhale, to visualize a balloon or cocoon of white light surrounding and protecting you. I had a bad allergic reaction - hives, itching, difficulty breathing - to my first chemotherapy. At the second chemo, they reduced the dose of my chemotherapy and gave me intravenous anti-allergy medications (Prednisone, Benadryl) and I had a less-severe reaction. Prior to my third chemotherapy, I thought to "pop my REBAL." I had no reaction to that particular therapy, and [the doctors] were able to resume full-strength chemotherapy and eliminate my pretreatment allergy medication after that. 


\section{About Hemi-Sync ${ }^{\circledR}$}

Through its research initiatives, The Monroe Institute (TMI), Faber, Virginia, has found that specific sonic frequencies can be combined to direct the brain gently into various states, from deep relaxation, or sleep, to expanded awareness and other "extraordinary" states. This "audio guidance" is achieved by sending a different tone to each ear through stereo headphones. The two hemispheres of the brain then act in concert to "hear" a third tone-the difference between the two tones. The third tone is not an actual sound, but an electrical stimulus that can only be perceived within the brain as a result of both brain hemispheres corresponding simultaneously. This results in a focused, whole-brain state known as hemispheric synchronization, or "HemiSync"-a process that optimizes human performance.

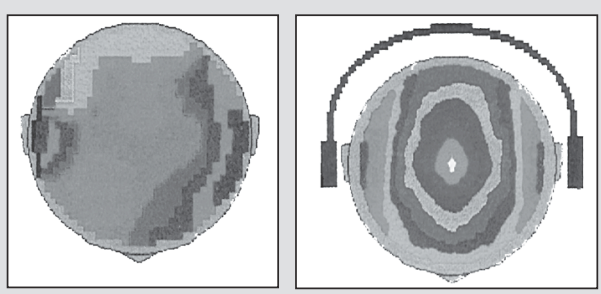

Map of the brainwave patterns in a normal brain, before (left) and after (right) exposure to Hemi-Sync. ${ }^{\circledR}$ The brainwave activity shifts from a random pattern with a limited focus to a highly coherent, well-focused whole-brain pattern. Scans reproduced with permission of The Monroe Institute, Faber, Virginia.

The patented Hemi-Sync ${ }^{\circledR}$ process has been refined via more than 40 years of research and development. Ongoing experimentation, data collection, and analysis are conducted at TMI's laboratory facilities to demonstrate the correlation between subjective experiential reports and objective scientific validation.

Ongoing research is essential to verify the influence of new, specific Hemi-Sync sound patterns on human consciousness. Over the decades, these efforts have resulted in the development of more than 200 individual programs for specific applications, such as focused attention, stress management, meditation, sleep enhancement, and pain management, to name a few. New Hemi-Sync programs are continually in development, refinement, and testing. For information on the full line of Hemi-Sync ${ }^{\circledR}$ CDs and cassettes, contact:

\section{Monroe Products}

P.O. Box 505

Lovingston, VA 22949

Phone: $800-541-2488$ or $434-263-9692$

Fax: 434-263-8699

e-mail Interstate@Hemi-Sync.com

Web site: www.Hemi-Sync.com

Disclosure: Brian Dailey, M.D., F.A.C.E.P., is a member of the professional division, the Dolphin Energy Club, and the Board of Advisors of The Monroe Institute. He receives no income from TMI and receives no income from the sale of TMl's products.

encing it, returned to his temple with a large number of HemiSync exercises. He felt the younger monks could learn-in 1 week-what had taken him years of meditative practice.

RM: What happened next?

BD: Shortly thereafter, I went down to TMI and took two 6- day courses, Gateway and Guidelines, back to back. It was a monumental growth period for me.

\section{RM: Why was that?}

BD: Each 6-day residential course takes place in the Blue Ridge Mountains on the grounds of TMI, sharing meals and the experience with the others attending. There are numerous Hemi-Sync exercises that one experiences in a progressive manner, each expanding on the previous experience. TMI attracts an incredibly interesting mix of people and healers.

More than half of the healing techniques I have learned have occurred while I was there. Karen Malik, president of International Society for the Study of Subtle Energies and Energy Medicine (ISSSEEM), and a trainer at TMI, took time to introduce me to some crystal healing techniques that I now use every day. It's one of the most enlightening experiences I have ever had and has made me a much more effective physician; not just in allopathic medicine, but in other healing arts, such as Reiki, Therapeutic Touch, ${ }^{\mathrm{TM}}$ CranioSacral Therapy, crystals, and aromatherapy.

RM: How long after taking these sessions did you begin to apply what you had learned into your medical practice?

BD: Immediately. I was applying it there at TMI as I learned it. Applying the techniques to my patients back home was a natural extension. Using the technique in my practice brought immediate and tangible results for my patients.

RM: What do these recordings actually do to the individual who uses them?

BD: Hemi-Sync is short for hemispheric synchronization. Sounds embedded on the CD result in a "binaural beat" that is perceived by the brainstem. A "frequency following response" results in both hemispheres becoming synchronized into a whole brain state that allows a user to simultaneously access both the creativity and intuitive insight of the right brain with the analytic insight of the left brain.

Many cognitive states that support healing, manifestation, and problem solving may be facilitated, depending on the specific frequency patterns that are used. Different frequencies will produce different effects. Theta and delta frequencies help with relaxation and sleep. Beta frequencies increase mental clarity, concentration, and produce a laser-like focus, which students find useful for studying, and children with attention-deficit disorder/attentiondeficit hyperactivity disorder find helpful with concentration.

RM: Please explain how this sound technology can assist patients who have cancer.

BD: We have found anecdotally that Hemi-Sync frequencies, along with the positive guided imagery on the CDs, can reduce adverse effects of such cancer therapies as chemotherapy and radiation. The technology may decrease neutropenia and anemia, reduce or eliminate nausea and vomiting, and improve overall well-being. All it takes is 45 minutes and a portable CD player with headphones. We are currently developing protocols to do formal studies of the Cancer Support Series. 
RM: Please explain the concept of "reframing" in the Cancer Support Series.

BD: As we know, the mind has a tremendous influence on healing. Many of the Hemi-Sync programs may enhance or "reframe" conventional viewpoints, as the Cancer Support Series does. Many people, particularly those with a holistic view, consider chemotherapy to be a poison. Chemotherapy has many potential side-effects, such as nausea/vomiting, diarrhea, hair loss, anemia, and neutropenia.

If a patient believes he or she is going to be pumped full of poison, how will that effect the healing response? I do not want my subconscious mind, let alone my conscious mind, to think such negative thoughts. Therefore, on the Cancer Support Series CD for Chemotherapy (which I helped to develop), in our guided imagery, we refer to the chemotherapy treatment as a "Love Potion." We refer to chemotherapy as a love potion because it is used to make the person well, not ill; and that needs to be understood, appreciated and embraced. Can you appreciate and feel the difference between taking a love potion to make you well and a poison?

RM: You mentioned crystals as healing enhancers. Please explain your use of crystals and how they relate to your practice of energy medicine.

BD: Each crystal has a frequency of vibration. Quartz crystals vibrate at 60 cycles per second, which is why quartz is used as a timing mechanism in watches. If you take two tuning forks that are tuned to a $\mathrm{C}$ of $128 \mathrm{~Hz}$, held next to each other, and strike one so that it vibrates, the other will start vibrating because of the principle of resonance.

Crystals may be used to store energy and stimulate the human body; this works according to the principle of resonance, or "vibrational medicine." 2 Healing may be done without crystals but I truly enjoy their feel and energy, as do many of my patients. Crystals will amplify energy and can be used to direct or focus healing energies. Crystals may be placed on specific chakras to help amplify healing energy.

Mitch Gaynor, M.D., has described the use of quartz crystal bowls to produce sound useful in healing cancer. We use quartz crystal bowls along with Hemi-Sync in our Reiki circles. It elevates the energy of the entire room as well as the energy of all the people present.

RM: Many allopathic physicians and ACM practitioners are striving to bridge the gap that exists between conventional and alternative medicine. You seem to be doing this now; please tell us your thoughts about this.

BD: I look forward to the day when we no longer consider them "conventional" and "alternative" forms of medicine but can appreciate that we have therapies of any modality that will help patients. I believe the placebo effect is a very real energetic effect that we have been unable to measure and characterize properly. We are on the cusp of the next great adventure-how energy medicine and human consciousness can affect healing, and how, consciously, we may use these energies to make us well.

RM: What sparked this integrative view-that is, using a combination of protocols, such as Reiki, crystals, and the Hemi-

\section{About The Monroe Institute}

The Monroe Institute (TMI)

356 Roberts Mountain Road

Faber, VA 22938

Phone: $866-88 I-3440$ or $434-36 I-1252$

Fax: 434-36I-I237

e-mail: Monroelnst@aol.com

Web site: www.monroe-institute.org

Thousands of people have attended TMl's various life-enhancing residential programs in the Blue Ridge Mountains of Virginia. These programs, conducted at the Institute's training facilities, are dedicated to developing, exploring, and applying expanded states of consciousness via Hemi-Sync ${ }^{\circledR}$ sound technologies.

Visit the TMI Web site for more information on workshops.

\section{Sync CDs-for you?}

BD: My father was a great influence. When I was 4 years old, my father taught me how to grow crystals, which I now use in healing. My mother had conventional medical training in nursing but, in addition, she was a great touch healer. If we skinned our knees, she "would rub it and make it better." If the hurt was really serious, she would "kiss it and make it better." And it always made us feel better. I was too young to understand that it was energy medicine, but I learned that one could make people feel better through touch, that it was a normal part of life.

\section{RM: What is the Dolphin Energy Club?}

BD: The DEC has a membership of 350 healers, coordinated by TMI, who attempt to heal and assist others from a distance using guided imagery, Hemi-Sync, and energetic healing. We ask recipients to record their impressions and results. It is clear that some people perceive benefits from remote healing. In the research lab at TMI, they did EEG brain mapping of healers while they did healing. A remarkably consistent brain map was found among many healers, and a Hemi-Sync tape was produced to induce this brainwave state. New members of DEC receive the DEC Hemi-Sync tape that is of benefit to all members, but particularly those with an interest, but no previous experience in remote healing. It is a wonderful gift to participate in DEC.

RM: Although ACM protocols have been slowly entering standard medical practice, many allopathic practitioners, and insurance companies such as health maintenance organizations [HMOs], remain unconvinced. What do you say to them?

BD: Delores Krieger Ph.D., R.N., has shown that Therapeutic Touch can increase hemoglobin blood levels in patients. This may be helpful for patients on dialysis and thoses with cancer who may be anemic or neutropenic as a result of their illnesses or therapies.

Fortunately, Epogen ${ }^{\circledR *}$ and Neupogen ${ }^{\circledR *}$ injections may be given to these patients to help improve their blood counts, at at cost of approximately $\$ 800$ for both injections.

Some of our patients who are using energetic techniques have been able to decrease the frequency of these injections, and in rare instances, have been able to stop them altogether. This can 
result in significant cost savings. Ironically, if you want the insurance industry to cover a specific therapy, you need to show insurers how it saves them money not just improves patient care. Reiki therapy is now partially reimbursed or discounted by HMOs in Rochester, New York. And, in the case of the Hemi Sync technology, one can buy a CD that is reusable for $\$ 20.00$ and perhaps reduce or maybe even eliminate the cost of some drugs used for diseases such as cancer.

RM: How do your medical colleagues react to your use of energetic techniques?

BD: My colleagues have been very supportive as they learn about these therapies. Rochester General Hospital had an ACM teaching day for its internal medicine residents. We had five Reiki tables, along with Hemi-Sync recordings; and we had 50 physicians participate-giving treatments as well as receiving them. The chief resident had chronic hip pain, which had caused her to stop running (her favorite exercise). She called 5 days later to say, excitedly, that, after a 15-minute treatment, she had no pain and had resumed running! She now works in private practice and has referred patients to me.

RM: Do you recommend that patients use headphones for maximum effect.

BD: Speakers placed on either side of the head will work fine but I especially prefer headphones for the first few listening experiences, as they screen out ambient noise and enhance the experience. The frequencies are like training wheels on a bicycle-after listening a few times, one can induce the experience at will.

*Both products are manufactured by Amgen, Inc., Thousand Oaks, California.
RM: Would you please clarify that? Can one do that without a CD playing?

BD: Yes, because once a person's brain becomes acclimated to the subtle synchronization energies, one can do it on one's own. Many of the Hemi-Sync exercises have encodings, or anchors, that are used during the exercise, so that one can recreate the effect later without the CD.

With the Chemotherapy Companion, if a patient becomes nauseous or vomits, the patient would inhale, and then, while exhaling, think "Plus-stomach calm." It induces the wonderfully pleasant experience the patient had earlier and reduces nausea/vomiting. There are signals to reduce pain, et cetera. It is also interesting that this technology is just as effective at low listening levels.

RM: Is there any downside to the Hemi-Sync CDs, or adverse affects you have observed?

BD: I have never observed an adverse or untoward affect. Unlike some medications, sound is not physically addicting. It doesn't interfere with, and is a wonderful adjunct to conventional therapies. For the practitioner, this is is an additional tool that is easily used. I tell patients that, in the worst case scenario, "you might feel better." My personal experience is that it has helped me and my patients greatly.

\section{References}

1. Kliempt $\mathrm{P}$, et al. Hemispheric synchronization during anesthesia: A double blind randomized trial using audiotape for intra-operative nociception control. Anaesthesia, 1999;54:769-773

2. Gerber R. Vibrational Medicine: The \#1 Book of Subtle Energy Therapies. Bear \& Company, 2001.

To order reprints of this article, write to or call: Karen Ballen, ALTERNATIVE \& COMPLEMENTARY THERAPIES, Mary Ann Liebert, Inc., 2 Madison Avenue, Larchmont, NY 10538-1961, (914) 834-3100. 